\title{
Retraction Note to: Multiview Gait Recognition Based on Silhouettes \\ Generated after Shadow Detection and Removal Using Photometric Properties Method
}

Rohit Katiyar, K.V. Arya, and Vinay Kumar Pathak

\author{
Retraction Note to: \\ Chapter "Multiview Gait Recognition Based on Silhouettes \\ Generated after Shadow Detection and Removal Using \\ Photometric Properties Method" in: K. Deep et al. (Eds.): \\ Proceedings of the International Conference on Soft \\ Computing for Problem Solving (SocProS 2011) \\ December 20-22, 2011, AISC 131, \\ https://doi.org/10.1007/978-81-322-0491-6_36
}

The Editors have retracted this chapter [1] as it contains sections that substantially overlap with the following articles (amongst others) [2-5]:

Co-authors K.V. Arya and Vinay Kumar Pathak stated that they were not involved in preparation of this manuscript, were not aware of its submission, and should not have been listed as authors.

All authors [Rohit Katiyar, K V Arya and Vinay Kumar Pathak] agree to this retraction.

\section{References:}

1. Katiyar R., Arya K.V., Pathak V.K. (2012) Multiview Gait Recognition Based on Silhouettes Generated after Shadow Detection and Removal Using Photometric Properties Method. In: Deep K., Nagar A., Pant M., Bansal J. (eds) Proceedings of the International Conference on Soft Computing for Problem Solving (SocProS 2011) December 20-22, 2011. Advances in Intelligent and Soft Computing, vol 131. Springer, India 
2. W. Kusakunniran, Q. Wu, H. Li and J. Zhang, "Multiple views gait recognition using View Transformation Model based on optimized Gait Energy Image,' 2009 IEEE 12th International Conference on Computer Vision Workshops, ICCV Workshops, Kyoto, 2009, pp. 1058-1064. https://doi.org/10.1109/ICCVW.2009. 5457587

3. A. Leone, C. Distante, Shadow detection for moving objects based on texture analysis,Pattern Recognition, Volume 40, Issue 4, 2007, Pages 1222-1233, https:// doi.org/10.1016/j.patcog.2006.09.017

4. Ju Han and Bir Bhanu, "Individual recognition using gait energy image," in IEEE Transactions on Pattern Analysis and Machine Intelligence, vol. 28, no. 2, pp. 316-322, Feb. 2006. https://doi.org/10.1109/TPAMI.2006.38

5. K. Bashir, T. Xiang and S. Gong. Feature Selection for Gait Recognition without Subject Cooperation. In M. Everingham and C. Needham, editors, Proceedings of the British Machine Conference, pages 108.1-108.10. BMVA Press, September 2008. https://doi.org/10.5244/C.22.108 